\title{
Urgences
}

\section{Neige noire de Hubert Aquin et Hamlet de Shakespeare, ou le sens de la rivalité}

\section{Jacquelin Marcheterre}

Numéro 25, octobre 1989

Multiples de Hamlet

URI : https://id.erudit.org/iderudit/025544ar

DOI : https://doi.org/10.7202/025544ar

Aller au sommaire du numéro

Éditeur(s)

Urgences

ISSN

0226-9554 (imprimé)

1927-3924 (numérique)

Découvrir la revue

Citer cet article

Marcheterre, J. (1989). Neige noire de Hubert Aquin et Hamlet de Shakespeare, ou le sens de la rivalité. Urgences, (25), 54-67. https://doi.org/10.7202/025544ar d'utilisation que vous pouvez consulter en ligne.

https://apropos.erudit.org/fr/usagers/politique-dutilisation/ 


\section{Neige noire de Hubert Aquin et Hamlet de Shakespeare, ou le sens de la rivalité Jacquelin Marcheterre}

Prise dans le champ plutôt vaste des études comparées, la lecture envisagée ici entend souligner tout simplement l'être hypertextuel de Neige noire ou, si l'on préfere, l'affleurement insistant de l'hypotexte shakespearien; elle entend accentuer l'effet de contraste de la pratique transpositionnelle opérée par Hubert Aquin. Pour ce faire, elle empruntera les avenues ouvertes par la réflexion théorique de Gérard Genette.

Mais au moment d'entamer Neige noire, de toucher ses revers palimpsestueux, une mise en garde: se méfier toujours du vertige lectoral que suscite la pratique hypertextuelle (quelque peu ambiguë) de Hubert Aquin.

Le texte s'ouvre sur une suite d'indications scéniques qui sont elles-mêmes investies, comme si l'impact de la forme didascalique ne suffisait pas, par certains éléments propres au discours métatextuel. Déjà, Neige noire engage une réflexion inattendue sur le texte fondateur. Le rideau se lève sur la scène montréalaise, dans la chaleur du mois de juillet 1973, chaleur tout à fait insupportable, qui plonge la ville et la population entières dans une sensation d'étouffement. Le climat évoqué ici n'est pas celui de la terrasse du château (sur les remparts d'Elseneur, pendant le guet...) où l'âpre froid ne cesse de gêner la garde de Francisco: il fait bien plutôt figure de contraste. Il suffit sans doute de retenir, pour l'instant, le caractère (ou la valeur) oppositionnel(le) de la transformation effectuée. Dès l'entrée du texte, le renversement de la température (elle passe du très froid au très chaud), d'ordre thématique, signale fortement le déploiement (à venir) de tout un " langage rival " 1 , suivant, d'une façon très adroite, le modèle du paradoxe d'inspiration nietzschéenne ${ }^{2}$.

Il y a, en fait, de nombreuses didascalies dans Neige noire: elles se révèlent parfois très longues et souvent (hyper)

1 On abordera la notion de rivalité plus en détail dans la deuxième partie de la présente étude, puisqu'elle s'illustre davantage sur le versant thématique de Neige noire.

2 Marie-Claire Ropars-Wuilleumier, “Le spectateur masqué. Étude sur le simulacre filmique dans l'écriture d'Hubert Aquin ", dans Litterature, Paris, $n^{\circ} 6: 3$, octobre 1986, p. 52. 
détaillées; tandis que dans Hamlet, les didascalies, plutôt rares, se bornent simplement à évoquer le décor, à désigner tel personnage, etc. Neige noire, bien loin de remettre en cause la qualité ou l'impact de la forme didascalique, traduit d'une manière fort pertinente le rapport exigeant qu'elle entretient déjà avec la technique de la description.

Le dialogue, lui, se compose de deux types de répliques bien distincts: l'un caractérise les personnages de Hamlet, l'autre les personnages aquiniens.

Il est un exemple frappant, à notre avis, d'une séquence où l'un et l'autre types de répliques consentent, en un même lieu textuel ${ }^{3}$, à s'imbriquer de façon admirable. À vrai dire, l'émission télévisée de Hamlet (image cadrée d'un téléviseur, métaphorique, au sens fort, en regard du récit) devient littéralement le théâtre d'un montage d'extraits: elle compose un regard critique, télévisuel, exercé "à distance", étymologiquement, sur la tragédie de Shakespeare, regard qui autorise une succession et, du même coup, une sélection de répliques entrecoupées. Le cadre cognitif (la manière dont ce cadre est utilisé pendant la séquence) provoque un glissement, une réorganisation critique, dans la mesure, justement, où le montage, enchâssé à son tour dans le fil du récit aquinien, concourt à ébranler le statut "classique" des répliques shakespeariennes au profit d'une contamination scripturale, du reste fort moderne, qui allie la tragédie à la fragmentation. Le montage semble donc acquérir, par lui-même, une portée proprement hypertextuelle; tout en reconduisant Hamlet, à tout le moins par fragments, le montage constitue une manière de produire autrement le "contenu " de la pièce shakespearienne: il représente peut-être, de par sa forme et suivant son insertion particulière dans la trame du récit, l'actualisation même de la modernité de Hamlet ${ }^{4}$.

Enfin, il y a, outre les didascalies, cette suite réflexive ponctuée d'indications scéniques, et le registre dialogique constitué principalement des deux types de répliques, un

3 Le lieu textuel dont il est question se trouve entre les pages 174 et 185 de la dernière édition de Neige noire (Montréal, Cercle du livre de France, 1978).

4 Voila tout de même une pratique particulierement perverse en ceci qu'elle préserve les répliques hamlétiennes intactes, répliques qu'elle détourne, par la même occasion, de leur lieu textuel d'origine: transplantation pour bien dire. Lesdites répliques se retrouvent hors contexte avec les avantages et les inconvénients que cela comporte. 
56

troisième niveau d'écriture, le discours parenthétique: "série de textes entre parenthèses ( 56 au total) qui constituent une succession d'impressions, de commentaires et d'analyses " ${ }^{5}$. Il suffirait d'évoquer, à titre d'exemple, la clausule de Neige noire pour mettre en relief l'importance de cette stratégie du texte qui privilégie, encore ici, le discours métatextuel, lequel n'apparaît pas dans Hamlet, du moins pas sous cette formelà. Métatexte aquinien dont la principale fonction semble être celle de mettre en relief le métadiscours shakespearien, plus profondément enfoui dans la lettre de la pièce, et qui nécessite bien, lui aussi, une attention particulière.

Tout cela pour dire que Neige noire participe alors d'une transposition purement formelle: la transmodalisation.

En effet, la transmodalisation réalisée ici - transformation portant «sur ce que l'on appelle, depuis Platon et Aristote, le mode de représentation d'une ouvre de fiction ${ }^{6}-$ témoigne d'un certain changement de mode: on passe du dramatique au narratif, d'une pièce de théâtre (de l'époque élisabéthaine) à un roman (du XXe siècle). Toutefois, puisque Neige noire préserve, en quelque sorte, le registre dialogique, le changement de mode ne peut être qualifié de radical; il ne va pas, par ailleurs, sans soulever quelques problèmes d'interprétation. Car ce roman est aussi un scénario. La narrativisation n'échappe guère, pendant la lecture, à la scénarisation, à la persistance du dialogue, qui est aussi le propre de l'écriture dramatique. À cet égard, le projet formel à partir duquel Neige noire affirme son être hypertextuel est tout à fait éloquent. On a l'impression de lire, de traverser une modulation du mode dramatique de la représentation, de saisir, sous le couvert d'une composition «romanesque», une variation theâtrale, dont la valeur scripturale, littéraire, tient sans doute à la façon ambiguë, perverse, suivant laquelle se déroule la critique de Hamlet (dans son ensemble) à travers l'élaboration d'une véritable poétique de la représentation théâtrale.

Cette variation théâtrale finit par détourner la lecture vers une meilleure compréhension de la fragmentation, au profit du pluriel de Hamlet, de son propre éclatement.

5 Pierre-Yves Mocquais, Hubert Aquin ou la quête interrompue, Montréal, Cercle du livre de France, 1985, p.146.

6 Gérard Genette, Palimpsestes. La littérature au second degré, Paris, Seuil, coll. "Poétique \#, 1983, p. 323. 
Voilà sans doute un bel exemple de transposition formelle - de transmodalisation inter- et intramodale - où le mode dramatique trouve une évaluation au plus fort d'une écriture résolument «moderne», là où la Forme ne cesse d'être remise en jeu, au risque même de s'y abolir.

Tenant compte de la relation de transformation unissant Neige noire et Hamlet, nous nous proposons maintenant de considérer la transposition au sens le plus fort, ouvertement thématique, qui touche à la signification même de l'hypotexte, que l'auteur de Palimpsestes nomme la «transformation sémantique".

C'est dire que, désormais, nous serons attentif à « la transposition diégétique, ou changement de diégèse, et [à] la transposition pragmatique, ou modification des événements et des conduites constitutives de l'action " 7 : couple de pratiques transformationnelles très étroitement reliées, dont la rentabilité semble, pour ce qui a trait à Neige noire, indéniable.

Rappelons que l'histoire racontée dans Neige noire se déroule d'abord à Montréal, puis se transporte à Oslo, en Norvège, et enfin dans les neiges noires du Spitzbergen. Au fil d'une quête éperdue, certains passages (qui sont autant d'analepses) rappellent au lecteur l'antériorité obsédante du hors-temps norvégien. Après la mort de Sylvie, tombée dans une crevasse, l'histoire se reporte de nouveau au Québec, suivant une sorte de translation spatiale qui la ramène à son univers originel. Lisible pour elle-même, l'histoire racontée dans Neige noire l'est aussi, bien sûr, en fonction de l'histoire représentée dans Hamlet, l'une étant le miroir, si l'on veut, de l'autre. Reflet par moment difficile à lire, à cause de l'apport signifiant du cinéma en particulier: entre la surimpression (l'effet de simultanéité) et le contrechamp (l'effet d'opposition), Neige noire instaure tout un langage qui oppose et relie hypo- et hypertexte. Ce langage est capable de contenir, de renfermer et de maîtriser des aménagements de structure dont l'intérêt transpositionnel ne se dément guère: ces aménagements ne sont certes pas inédits, mais ils sont provocants sur le plan

7 Gérard Genette, Palimpsestes, p. 341. On se doit, en plus, de lire la note que Gérard Genette ajoute au terme « pragmatique " : « Je dérive ici l'adjectif du grec pragma, qui désigne, chez Aristote et ailleurs, toute espéce d'événement ou d'acte: c'est en somme le sens courant, mais restreint à son champ proprement litteraire (l'action narrative ou dramatique). " 
sémantique, et d'une hardiesse surprenante en regard de l'hypotexte.

On constate alors, dans cet univers quasi cinématographique, que:

a) la surimpression ne va pas sans accentuer le brouillage, la confusion qui règne dans la sphère actantielle ${ }^{8} \mathrm{de}$ tel personnage. Un exemple: Sylvie, assimilée à Linda Noble, " comme si elles se cherchaient l'une l'autre" (Neige noire, p. 23), ne tarde pas à occuper de manière imparfaite: 1. la zone du dédoublement (lors de la parodie sacrificielle à l'intérieur de l'appartement de Stan Parisé); 2. la zone du redoublement (lors de sa tentative de suicide, tête première dans l'eau d'une baignoire de l'hôtel Bonaventure, à la manière d'Ophélie) ${ }^{9}$. De la confusion au désordre, il n'y a qu'un pas, bien entendu. Mais la confusion a certes plus à voir, en pareil cas, avec l'ambiguïté, avec la profusion ou, si l'on préfêre, avec l'abondance des avenues offertes à la lecture comparative ${ }^{10}$;

b) le contrechamp, par ailleurs, ne va pas sans accentuer la part d'opposition/ressemblance déjà présente dans Hamlet. Opposition qui règne aussi, par exemple, entre les personnages de Nicolas et de Sylvie. Par la mise en valeur de la prise de vue opposée, le contrechamp met au

8 La notion de sphere actantielle permet tout simplement de rendre plus évidente l'attraction d'un personnage concerné par la modification d'un événement, de mettre à l'épreuve, surtout, la force dramatique qui l'attire vers telle conduite.

9 Le principe du dédoublement n'a cours qu'à l'intérieur d'un même système; ainsi en est-il, par exemple, lorsque Linda Noble (personnage de Neige noire) endosse exactement le rôle de Sylvie Lewandowski, au moment où elle occupe sa place aupres de Nicolas lors de la parodie sacrificielle (dans l'appartement de Stan Parisé, le metteur en scène); dans une moindre mesure, lorsque Eva de Norvège se méle d'occuper la place de Sylvie, de jouer auprès de Nicolas un rôle cependant efficace et intimidant. Le principe du redoublement, en revanche, implique nécessairement la présence de deux systèmes; II en va ainsi lorsque Linda Noble joue le rôle d'Ophélie, personnage hamlétien, pendant la répétition théâtrale de Hamlet (dans les studios de Radio-Canada à Montréal); plus subtilement, lorsque Nicolas interprète le rôle de Fortinbras sur les ondes, lors de la représentation télévisée.

10 lci, le mot " confusion " n'est pas utilisé péjorativement, mais plutót dans la perspective d'un projet d'écriture condamné par avance a la faillite, où la ruine des relations proprement actantielles fonde justement le développement de la scénarisation: tout le simulacre filmique dont parle Marie-Claire RoparsWuilleumier. 
jour un face à face actantiel 11 que l'histoire se charge d'exploiter, jusqu'à la limite du possible.

C'est dire le pouvoir qu'exerce le visuel sur le nominal ${ }^{12}$, qui doit pleinement son existence au changement de diégèse: ce nouvel univers ne cesse de relancer, en vertu de ses continuelles références au cinéma (et à la connaissance technique de cet art), une histoire qui n'est plus de Hamlet tout lui restant, de façon paradoxale, très fidèle; une histoire retournée quelque peu sur elle-même, dont les événements ont été sérieusement modifiés par le passage d'un univers à l'autre.

Il importe donc de saisir la transposition pragmatique pour elle-même, dans ce qui constituera le volet le plus étendu de cette étude.

Autant se remettre tout de suite en mémoire la rencontre d'Eva et de Michel Lewandowski, au restaurant Saint-Malo, peu de temps après la représentation télévisée de Hamlet. Ils discutent la mort de Sylvie. Plans intercalés, rapides, de Sylvie. Le lecteur est mis au courant, par diverses insertions analeptiques, de la relation que Sylvie entretenait avec Michel Lewandowski. [...] Nicolas et Eva font du repérage, sur l'île des Sœurs, en vue d'un film. Image très brève de Sylvie, de retour de New York, et de Michel, au terminus de la gare centrale de Montréal. Nicolas fait part de ses appréhensions quant à la réalisation de son film, à propos, surtout, de sa vérité sur le strict plan autobiographique. Autre image de Sylvie dans les bras de Michel Lewandowski: le lecteur apprend, enfin, la véritable nature de la relation qu'elle entrenait depuis longtemps avec nul autre que son père. Autre plan. Sylvie et Michel font l'amour pour la première fois. Ils se disent leur désir commun, ils veulent demeurer, malgré tout, heureux. [...] Nicolas, en passe de terminer le repérage, fait plusieurs plans de la maison de Michel, qu'il trouve splendide, ne sachant pas alors qu'elle a été le lieu et le théâtre des amours du père et de sa fille.

Voilà pour le synopsis du récit.

11 La vertu transpositionnelle du contrechamp permet ainsi de acontrer" le sens immédiat de l'hypotexte: il s'agit d'une façon littéraire d'inverser la thématique hamlétienne, de s'opposer (du moins partiellement) à la répartition initiale des róles, à leur force dramatique.

12 Voir à $œ$ sujet: "La dialectique du visuel et du nominal " dans Hubert Aquin ou la quête interrompue de Pierre-Yves Mocquais. 
60

Il y a, à l'origine, transgression, rupture de l'ordre: l'inceste de Sylvie et de son père, Michel. Dans Neige noire, Sylvie est la cause d'un désordre familial auquel d'ailleurs Nicolas n'échappe guère, pris au piège lui aussi, dans l'engrenage de l'action. La relation incestueuse de Sylvie et de Michel correspond, bien sûr, à la relation troublante de Hamlet et de sa mère, Gertrude. Renversement des genres, chiasme donc, rapports de force inversés. Mais, sous un autre angle, l'inceste de Sylvie, de par son antériorité en regard du temps du roman, fait écho à l'assassinat de Hamlet père. Claudius attente à la vie du roi, il le tue et, du coup, il ébranle l'État et ses loyaux sujets; ébranlement qui sera le "royaume", désormais, de Hamlet fils. Claudius provoque, par là, une rupture qui est antérieure, elle aussi, au temps de la diégèse, il brise l'harmonie de l'État avant même que l'histoire de Hamlet ne débute. Dans Neige noire, Sylvie provoque, au niveau de la cellule familiale, un remous tout à fait comparable. À la seule différence qu'il y a, dans le cas de Sylvie, inceste au lieu de meurtre ${ }^{13}$.

llgne agressive

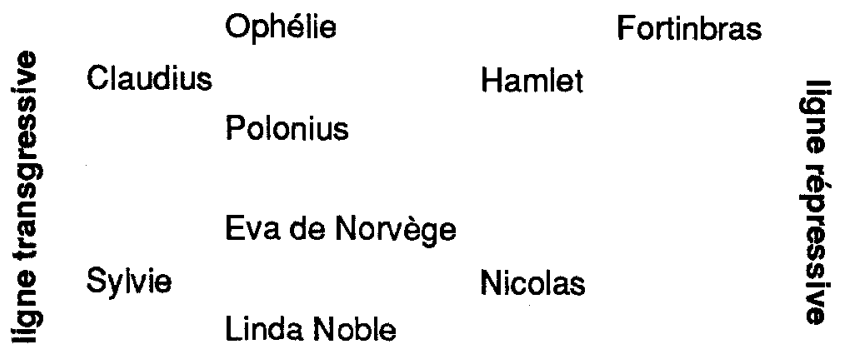

ligne agressive

En qualité de meurtrier, Claudius se trouve donc à la lisière de la ligne transgressive, qu'il partage avec Sylvie, nécessairement coupable d'inceste. Mais si, d'emblée, on s'attend ici à voir apparaître Sylvie sur la ligne transgressive, il est plus difficile de montrer en quoi sa conduite relève d'une

13 Afin de pallier le risque d'égarement, on retiendra un carré relationnel sur lequel on peut déjà lire, a priori, une rivalité dramatique représentée d'ailleurs suivant trois lignes de conduite (agressive, répressive et transgressive) que nous mettrons au jour en cours d'analyse. 
transposition sémantique. Il y a, au départ, refus, désobéissance. Sylvie ne se conforme pas au système établi, elle repousse l'interdit qui pèse sur la relation de nature incestueuse, à moins qu'elle ne s'efforce de le nier tout simplement. L'écart de conduite reste pourtant entier. Sylvie couche avec son père. Pour sa part, Claudius se rend coupable de désobéissance à l'égard de la loi constitutionnelle du royaume; insoumis, il défie le trône, il tue perfidement le roi Hamlet. En ce sens, Sylvie «redouble» Claudius, c'est-à-dire qu'elle reproduit la nonconformité de sa conduite. Mais contrairement à Claudius, Sylvie perd peu à peu le pouvoir qu'elle exerce sur ses relations. La désobéissance conduit à la soumission. Sylvie est tenue de se soumettre aux désirs incestueux de son père. Cependant, la désobéissance dont elle se rend coupable dévoile, mine de rien, le motif à la base de la conduite de Claudius: à travers l'exercice du pouvoir, par-delà le plaisir de gouverner l'État, Claudius semble viser la couche incestueuse.

Ainsi donc, la conduite transgressive de Sylvie aurait pour effet de revaloriser l'acte incestueux, sinon de lui redonner toute sa force, subtilement occultée d'ailleurs dans Hamlet. Confronté à Neige noire, Hamlet trouve de nombreuses résonances; le dévoilement de l'inceste ouvre, eu égard au jeu des personnages en général et à la mise en place des événements dramatiques, de nouvelles voies interprétatives.

Un coup d'œil maintenant sur l'épisode du Spitzbergen.

Dans la hutte, tout est calme; dehors, une chute de neige abolit toute vision, toute réflexion. Nicolas déshabille Sylvie, après le repas, devant le feu. Coupure. Dans le blanc de l'interligne, Nicolas exécute sa vengeance; il tue Sylvie suivant une sorte de rituel ${ }^{14}$. Leurré par les multiples interruptions du récit, le lecteur n'apprend les circonstances de la mort de Sylvie que beaucoup plus tard. Dans l'appartement de la rue Berri, Nicolas, maîtrisé, dominé par Eva, finit par avouer son meurtre: l'aveu arraché dans la nudité ${ }^{15}$, sous la torture du

14 Pierre-Yves Mocquais élabore de très belles pages sur "le rituel dionysiaque et la poursuite d'une catharsis idéale " dans Hubert Aquin ou la quête interrompue.

15 Le dénudement du corps ne cesse de mimer, sur le mode allégorique, celui, toujours plus pressant, de la vérité restée masquée, jusque-là, par le mensonge fignolé. II faut remarquer, surtout, comment Nicolas glisse, progressivement et d'une manière lamentable, irréversible, de la dénudation physique à un profond dénuement moral. 
62

plaisir, renvoie à la façon plutôt sadique suivant laquelle il avait réalisé sa vengeance préméditée, méticuleusement préparée. Â nouveau, l'intérieur du refuge. Sur le Spitzbergen. Devant le feu de bois, Nicolas attache Sylvie: il assujettit ses jambes et ses bras. Sylvie a peur; elle crie à tue-tête. Sans trop tarder, Nicolas procède à une excision: il lui coupe le clitoris. Il lui fait une petite incision qu'il mène, toujours avec la même lame, de la vulve jusqu'aux seins. Puis, il lui entaille le visage, de chaque côté, et, juste au-dessus des yeux, les muscles du chagrin. Pendant ce temps, Nicolas lui reproche sa relation incestueuse; il lui confirme, sans peine, la haine qu'il entretient à l'égard de son père, Michel, haine du Père sans doute aussi, qu'il extrait en creusant, littéralement, le corps coupable de son amante. Nicolas transperce Sylvie avec sa lame, finalement, juste sous le sein gauche. Il l'emballe tout de suite dans la toile de la tente; il la glisse au dehors du refuge, sur la neige, jusqu'aux abords d'un précipice. Et de là, il la pousse, sans hésiter, dans le vide. Fondu au noir.

À l'opposé donc de la ligne de conduite transgressive, se trouve la ligne répressive: le personnage de Nicolas (amant et compagnon de Sylvie, mais, suivant la ligne agressive, opposant à la conduite incestueuse de sa maîtresse), véritable redoublement de Hamlet («héritier» de Claudius, mais, suivant la même ligne agressive, farouche opposant à sa politique étatique et familiale). Sous le coup d'une pulsion (maniaque et maitrisée), Nicolas prépare une vengeance forcenée, d'une rare intensité. Une vengeance tardive dirigée contre Sylvie. Une vengeance qui doit juguler l'étendue grandissante des dégâts incestueux: le mal psychologique et le désordre familial qui en résultent. C'est là une vengeance que traduit bien le dispositif d'écriture utilisé alors dans le récit: la descente dans l'abîme de Sylvie correspond, quant au moment où elle prend place dans le roman, en son centre ou à peu près, au moment où, dans Hamlet, a lieu la représentation mise en abyme. Mais alors que la représentation hamlétienne révèle tout, le roman, en son centre, tente de tout camoufler, de faire le vide, le silence.

En réalité, la problématique de la vengeance est intimement liée à celle, plus englobante, de la répression, dont la sanction corporelle infligée par Nicolas au personnage de Sylvie constitue le violent paroxysme.

Nicolas « redouble " bien la conduite répressive de Hamlet. À cette différence près que, contrairement à Hamlet qui 
s'évertue à libérer, par tous les moyens, le trône et le lit royal, pour s'y glisser sans doute souverainement, Nicolas, lui, s'efforce de préserver, de raffermir le rôle qu'il occupe auprès de Sylvie. Il est son amant et il veut être le seul à partager sa vie et son lit... À vrai dire, là où la conduite répressive de Nicolas se distingue de la conduite de Hamlet, c'est précisément dans le transport punitif. Hamlet ne tarde pas, d'ailleurs, à s'enflammer lorsqu'il apprend la vérité; il s'emporte bien vite à l'idée d'attenter à la conscience de Claudius; mais il se détourne pour regarder ailleurs, il dirige progressivement son action punitive vers la couche royale: il se porte, en dépit des avertissements de son père, dans les bras de sa mère. Hamlet blesse Claudius, il porte atteinte à son intimité, sur son propre terrain. Pour sa part, Nicolas, se venge plus furieusement encore, contre la conduite trangressive de Sylvie, il l'entraîne loin de son père, Michel; il gagne ni plus ni moins son pari en arrachant Sylvie à sa relation incestueuse: il l'emporte sur un tout autre terrain. Dans tous les sens du terme. Certes Nicolas ne vise pas, contrairement à Hamlet, la place ou le privilège du Père; mais il table bien plutôt sur l'autorité, la liberté tragique avec laquelle il peut jouer, désormais, un rôle d'un interdit tout aussi cruel. Un rôle susceptible de plaire à Sylvie, de la séduire, de la tromper par le mensonge. Un rôle qui lui permet d'entreprendre une mesure punitive telle que seule la mort, la violence ou le jeu, le rituel donc, peut racheter la trahison, dans l'isolement du Spitzbergen.

Nicolas et Hamlet, admis au premier chef sur la ligne répressive, commettent quand même des actes de nature transgressive. Nicolas, par exemple, considère l'inceste comme une menace qu'il faut d'abord contenir, un grave délit qu'il faut par la suite punir; il exerce alors de graves sévices à l'endroit de Sylvie; il la torture et finit par l'assassiner de sang-froid; il viole le droit fondamental à la vie et il se rend coupable, à cet égard, de transgression. Le principe est d'ailleurs valable pour la partie adverse. Sylvie et Claudius commettent, eux aussi, mais pour d'autres motifs, des actes de nature répressive. Claudius, par exemple, n'hésite pas à éloigner Hamlet du trône, à le déporter, au moment où il le juge nécessaire, vers l'Angleterre. Par là, Claudius tente d'étouffer la révolte, de punir, en quelque sorte, l'initiative de Hamlet afin de reprendre les pleins pouvoirs à la cour; il abuse du privilège royal, il viole le droit à la libre expression 
et se rend coupable, en vertu de la ligne de conduite adoptée, de répression.

Il n'est nul besoin d'autres détails ou de preuves supplémentaires pour comprendre que la logique décrite à partir du carré relationnel ouvre plusieurs avenues de rëflexion.

Outre les lignes transgressive et répressive, il reste néanmoins à analyser la ligne de conduite proprement agressive. La ligne agressive légitime, à elle seule, tout le déroulement de l'action dramatique de Neige noire; elle nuance, parfois même elle renforce ou elle "aggrave", la conduite de tel personnage hamlétien, elle justifie en tout cas le travail transpositionnel opéré. Une telle ligne favorise un couple, Sylvie et Nicolas, dont la conduite relève très précisément de la concurrence amoureuse; elle redouble, elle relance la conduite agressive de Claudius et de Hamlet, ce couple visiblement caractérisé par l'opposition guerrière. Voilà certes une distinction thématique qui, tout en précisant la nature de la transposition opérée (la revalorisation de l'affrontement hamlétien à l'intérieur d'une relation amoureuse perverse), confere à la pratique du redoublement, pour lors, un retentissement sémantique formel dont la vertu structurante est incontestable.

Effectuons donc, à titre illustratif, un retour sur l'agression de Nicolas. De l'aéroport de Dorval, l'avion décolle à destination de la Norvège. À son bord, Nicolas et Sylvie observent le sol. Nicolas tient Sylvie dans ses bras. Le pendentif en argent que Sylvie porte à son cou est subitement taché de sang. Changement de décor, analepse: la chambre de Sylvie. Sylvie est tendue; elle est en colère. Nicolas la harcèle, il insiste, il veut connaître à tout prix le nom de son amant, l'âge de l'homme qu'elle a fréquenté. Il s'agit de Michel Lewandowski, 49 ans. Sylvie blesse Nicolas au pénis avec le pendentif. Elle pleure, elle ne cesse de pleurer. Dans l'avion, Nicolas, penché sur Sylvie, est fasciné par le bijou. La chambre de Sylvie, de nouveau: dans un mouvement de colère à peine contenu, Sylvie blesse Nicolas en reprise; Nicolas s'essuie avec des tampons stériles. À l'intérieur de l'avion, Nicolas se réveille auprès de Sylvie, il file aux lavabos, il se rase.

L'agresseur, c'est de toute évidence Sylvie: elle émascule Nicolas au moment où il s'y attend le moins; elle lui rappelle, de la sorte, la vulnérabilité de son corps et, du coup, l'inconfort de sa position. À l'instar de Claudius, Sylvie adopte une 
conduite déloyale; elle ravit à Nicolas, en traîtresse, le pouvoir "viril" de la domination: elle brise momentanément son règne. Cependant, elle n'agit pas seule. À preuve, l'arme dont elle se sert, le pendentif en argent, porte en médaillon la photographie de Michel. Subtilement dissimulée par le récit, se profile la figure du Père, écho perverti, plus «moderne» et moins fantastique, de la représentation spectrale du père de Hamlet.

La force de frappe de Michel Lewandowski est d'autant plus grande qu'il est le personnage le plus mobile du récit. Il en va de même, d'ailleurs, dans le cas du père de Hamlet, qui a le don d'apparaitre toujours au bon endroit et de se faire entendre au bon moment. Michel Lewandowski assure son autorité par sa non-absence; il perturbe la vie de Nicolas en lui remémorant l'acte à punir sans jamais se manifester auprès de lui, sinon par l'entremise de Sylvie. Autrement dit, il est là sans être là. En revanche, le père de Hamlet renforce son pouvoir par sa non-présence; il perturbe la vie de Hamlet en lui rappelant la vengeance à accomplir, il se manifeste auprès de lui par la voie mystérieuse de l'apparition fantomatique: sans être là, il demeure sans cesse là. Plus remarquable encore est le passage de Michel Lewandwski de la nonabsence à la plus totale absence, puisqu'il se suicide. Le père de Hamlet, au contraire, passe de la non-présence à la présence puisqu'il reprend plus ou moins, des mains de Claudius mort, une part de la paix ou du pouvoir perdus; il acquiert, dès l'entrée triomphale de Fortinbras dans le royaume, le droit au repos de l'esprit.

La concurrence amoureuse (la rivalité, la rencontre des forces rivales) forme une ligne interactive où Sylvie apparaît, par exemple, dans un rapport d'opposition de nature substitutive. Sylvie, pourtant opposée de manière symétrique à la conduite de Hamlet, joue néanmoins, au moment où elle agresse Nicolas, un rôle vengeur: elle concourt à la revanche du Père. Révoltée, Sylvie exerce une fonction répressive, comme Hamlet: elle punit Nicolas. Elle exerce des représailles momentanées, brèves, mais efficaces dans la mesure où la voie de la riposte confirme justement sa présence et son engagement au sein d'une véritable guerre d'usure. Mais Sylvie perd rapidement la maitrise d'une situation qui la mène dans une impasse dont elle ne sort pas vivante. De même, Nicolas apparaît dans un rapport d'opposition de 
nature substitutive à Claudius lor squ'il commet le meurtre de Sylvie. À long terme, fatal pour Claudius (puisqu'il est éliminé finalement du champ d'action), le meurtre se révèle plutôt humiliant pour Nicolas (puisqu'il est facilement éloigné du champ d'action) ${ }^{16}$. À la fois lâche et vengeur devant la conduite de Sylvie (doublée, au retour à Montréal, par Eva de Norvège), Nicolas perd peu à peu la maîtrise de soi: dans un aveulissement semblable à celui dont est victime Claudius au sortir de La souricière, Nicolas finit, lui aussi, par dévoiler son horrible crime. C'est à partir d'un écart transgressif que s'échafaude et se construit pour Nicolas l'univers déroutant de la tragédie.

Une telle interaction autorise, en tout cas, la lecture de la concurrence amoureuse (redoublement de l'opposition guerrière hypotextuelle) sous un tout autre angle; elle active et réactive la fameuse figure du chiasme (présente techniquement dans le champ-contrechamp cinématographique) qui subsume en quelque sorte les qualités substitutives du carré relationnel de départ.

Déjà occupé par Claudius et Hamlet, à peu près pour le même motif ("avoir raison sur l'autre»), et par Sylvie et Nicolas, le carré appelle bien sûr d'autres personnages susceptibles de se greffer logiquement autour de ces sphères actantielles. Il peut être profitable de remettre le carré à l'épreuve, de réfléchir alors sur la nature et les implications agressives d'un autre couple, Eva de Norvège et Nicolas. Eva fait une brèche dans le projet d'écriture cinématographique de Nicolas; elle l'attaque de manière à combler le fossé, la fosse qui la sépare de Sylvie. En vue de lever le voile sur les circonstances de l'«accident», de les mettre à jour. À ce titre, la conduite d'Eva de Norvège s'apparente à la conduite inquisitrice de Fortinbras. Aux côtés de Hamlet (par la force des choses mais pour d'autres raisons «officielles"), face à

16 Le champ actantiel est tout simplement désigné par l'espace textuel dans lequel peut œuvrer un ou plusieurs personnages: Neige noire eri constitue un, Hamlet un autre. De cette manière, il y a, à l'intérieur d'un champ actantiel donné, présence de plusieurs sphères actantielles. Les sphères de Sylvie et de Nicolas se trouvent, par exemple, dans le champ actantiel de Neige noire et n'appartiennent qu'à celui-ci, alors que les sphères de Hamlet et de Fortinbras (dont les rôles sont endossés, à la suite ou en même temps, par Nicolas) se trouvent dans le champ de Neige noire mais appartiennent aussi, qu'on le veuille ou non, au champ actantiel de Hamlet. 
Claudius donc, Fortinbras ne tarde pas à traverser les territoires du Danemark: il s'immisce progressivement, sous le "prétexte polonais ", dans les affaires du royaume. Tout cela en vue de forcer les marches du trône, de recouvrer le pouvoir ancestral perdu par son père aux mains de Hamlet père. En retour, la conduite conquérante de Fortinbras dit déjà admirablement les mesures agressives prises par Eva: tous deux sont unis sous l'insigne victorieux de la Mère Norvège. Comédien, donc fondé par le jeu, Nicolas tente de puiser, dans le rôle de Fortinbras (source d'inspiration et de méconnaissance), toute la patience tactique de son intervention qui repose entre autres sur la rigueur interressée de la répétition. Â cette différence près qu'il échoue sur toute la ligne: il rate la répétition (lors de la parodie sacrificielle, en compagnie de Linda Noble) et il ratera son entrée au cinéma. Loin de mettre dans sa démarche la rigueur de Fortinbras, Nicolas prépare d'instinct le terrain de la vengeance; au lieu d'attendre patiemment la sortie du père (le suicide de Michel), il manœuvre sous le coup d'une impulsion qui le mène alors aux antipodes de ses visées initiales: il cesse d'écrire et il cesse en même temps d'exister.

Lire Neige noire en fonction du carré relationnel mis de l'avant ici conduit à cerner davantage la notion de rivalité, à accepter, pour lors, le modeste impact qu'elle crée sur l'objet de désir (essentiellement scriptural) qui fonde, depuis toujours sans doute, la pratique hypertextuelle: c'est-à-dire le passage et le contre-passage du sens, simultanément. Hypertextualité: lire, écrire et commenter tout à la fois. 


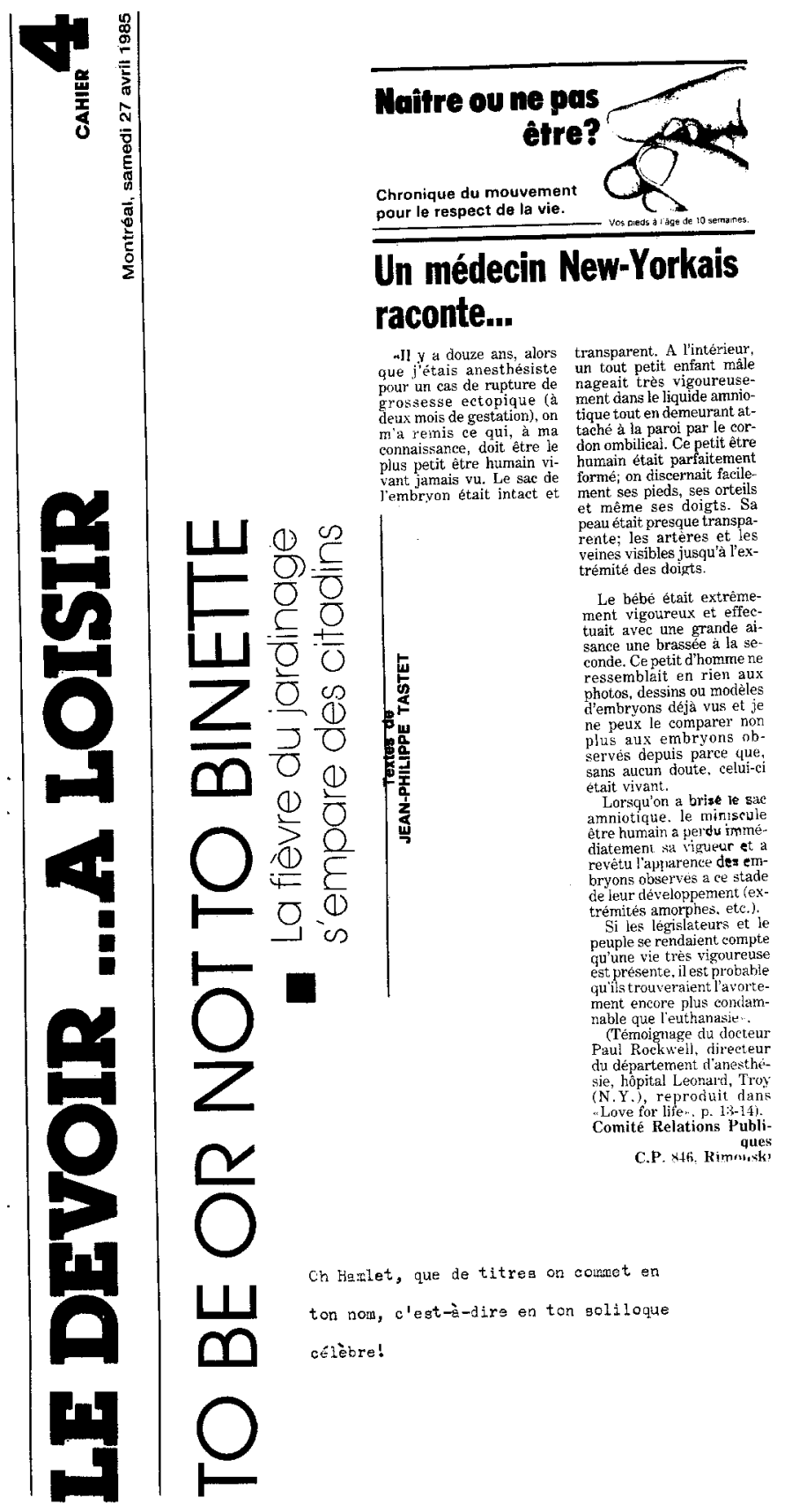

